\title{
Using Trust Game for Cross-Strait Security Risk Analysis
}

\author{
Cheng-Kuang $\mathrm{Wu}^{1, *}$, Chongfu Huang ${ }^{2}$ \\ ${ }^{1}$ Zhejiang Industry \& Trade Vocational College, Wenzhou 325035, China \\ ${ }^{2}$ Academy of Disaster Reduction and Emergency Management, Faculty of Geographical Science, Beijing Normal Uni- \\ versity, Beijing 100875, China
}

Received March 22, 2018

Accepted April 5, 2018

\begin{abstract}
The interactions of the Mainland China offering benefits, the Taiwan benefits return, and the benefits of ChinaTaiwan bilateral trust between the China and Taiwan are modeled as a trust game. The trust strategy is best response for China and Taiwan if the benefit of China-Taiwan bilateral trust is increased based on Nash equilibrium analysis. This study simulated a mixed strategy Nash equilibrium. Experimental results show that the China's expected payoffs of N.E. fix and Taiwan's expected payoffs of N.E. are increased if the payoffs of China-Taiwan bilateral trust are increased. The Chinese mainland released new measures on exchanges and cooperation with Taiwan. Taiwan accepts these measures results in favor of Taiwan's expected payoffs and decreases the level of risk.
\end{abstract}

Keywords: trust game, Nash equilibrium, bilateral trust, security risk

\section{基于信任博亦分析海峡两岸安全风险}

\author{
吴正光 ${ }^{1 *}$ 黄崇福 ${ }^{2}$ \\ 1. 浙江工贸职业技术学院信息与传媒分院, 浙江温州 325035, 中国 \\ 2. 北京师范大学地理科学学部减灾与应急管理研究院, 北京 100875 , 中国
}

\begin{abstract}
摘要：本研究应用中国大陆给台湾人民的利益、台湾回馈给大陆的利益, 以及两岸采用「信任」策略而产生的 合作报酬, 这三个互动因子建构非零和两岸信任博亦。从分析博弯的纯粹策略均衡中得知, 当双方采用「信 任」策略的合作报酬很高时, 这结果对双方是最佳的选择。现今两岸僵局正走在一个混合策略的信任博亦里, 经由数值模拟计算本模型的混合策略均衡后, 发现大陆的混合策略纳什均衡的期望报酬不会受到三个重要因子 影响而改变, 而台湾的混合策略均衡的期望报酬会随着两岸采用「信任」策略的合作报酬增加而增加，中国大 陆提出多项惠台政策，台湾接受这些惠台政策，才能提高台湾的混合策略纳什均衡的期望报酬，并降低两岸安 全风险。
\end{abstract}

关键词: 信任博亦; 纳什均衡; 互相信任; 安全风险

\section{1. 引言}

台湾地区的经济贸易发展曾经是亚洲四小龙之 一，这样的繁荣情景已经离台湾人很远了，反观近 几年中国大陆崛起，我们依据国际货币基金网站
（2017）发布 2005 至 2015 年中国及台湾地区的人均 国内生产总值（Gross domestic product per capita）， 建立年增长率比较图（如图 1)，图中可以看到中国 内地在 10 年里的人均国内生产总值的平均增长率为

* 作者简介: 吴正光 (1970) 男, 台湾桃园人, 毕业于台湾中央大学信息管理学系博士班, 助理教授, 长期从事博弯论应用 与数据分析之研究。E-mail: shapleyvalue@hotmail.com. 
$14.4 \%$, 而台湾地区为 $3.4 \%$ 。近四年两岸的人均国内 生产总值的增长率均下降, 而台湾地区在 2015 年时 呈现负 $1.2 \%$, 显示在 2015 年比较于 2014 年的人均 国内生产总值是呈现下降。Callen 学者 (2017) 指出 一个国家每年的国内生产总值 (Gross domestic product：GDP）提供了一个国家或地区的经济规模和表 现的重要信息。实际国内生产总值的增长率经常被 用来作为经济总体健康状况的指标。从广义来看, 实际 GDP 的增长被认为是经济表现良好的信号, 当 实际国内生产总值强劲增长时, 代表国内企业或工 厂雇用更多的工人，而且人们的口袋里有更多的 钱, 就业率可能会增加。Florence 等学者 (2016) 也 指出人均国内生产总值 (GDP per capital) 可以分别 由劳动生产率、劳动年龄人口和总人口的比率, 以 及就业年龄人口和总人口的比例三个部分所组成。 在 2007 至 2008 年全球经济危机期间, 当许多国家的 生产总值缩水时, 就业率就往下降。

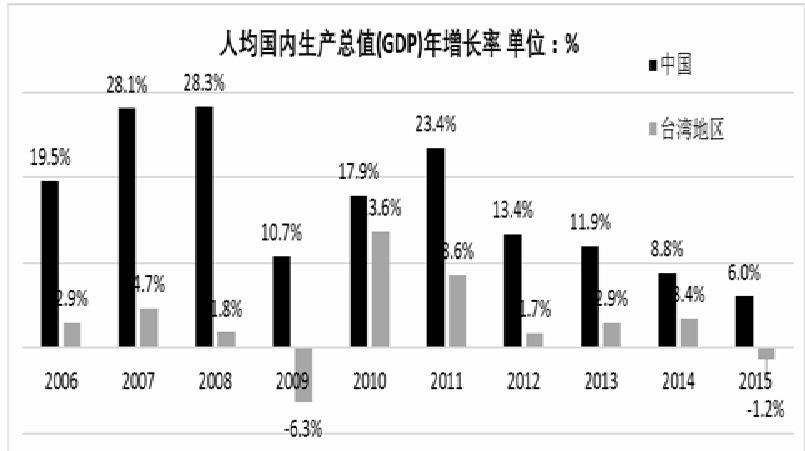

图 1: 中国大陆与台湾人均国内生产总值年增长率

Kastner 学者 (2015) 认为中国军力快速增长, 但海峡两岸密切的经济交流, 使得武装冲突的风险 一直在减少, 并可能在未来几年继续下降, 台海不 会再成为世界冲突事件的焦点（flash point）。文中 指出: 从长远来看, 中国深信, 两岸经济融合日益 加深及两岸人员往来增多时, 最终将有助于转变台 湾人认同一个中国的身份, 这将使两岸人民渐渐迈 向统一之路, 这样藉由两岸交流频繁比通过战争达 到统一得到的效果会更好。因此, 长期来看, 中国 领导者会放弃使用武力解决两岸问题。Raymond 和 Francois 学者 (2003) 研究两岸发生冲突时, 将中国 与美国的策略互动塑模在一个的树状博峦里, 他们 分析出中国对两岸的处理类型有四种: 第一, 对未 来两岸关系充满「自信」的中国是主张两岸和平, 而改善经济和政治关系以及建立两岸信任措施, 有 助于创造安全和平的环境, 这样的中国政治和经济 条件符合美国的利益。第二及第三分别是「有耐 性」和「没耐性」的中国, 两者都会视情况选择战 争解决两岸问题。美国在处理两岸关系时, 阻止一
个「没耐性」的中国比一个「有耐性」的中国更 难。在他们提出的博亦理论框架下, 除非武力统一 台湾成功概率很大，一个「有耐性」的中国才会发 动战争。然而, 一个「没耐性」的中国即使可能只 是成功概率很小, 也会发动战争完成统一。一个 「没耐性」的中国具有显着拒绝沟通的意识, 这一 点美国尤其难以遏制。还有对美国最糟的情况就是 第四个「对台湾人民绝望」的中国, 这样的中国一 定会发动战争完成统一, 台湾也没能力阻止。最 后, 他的博亦模型也说明, 美国要介入中国实现统 一发动的战争, 在台海应急投入的兵力资源, 回报 的利益并不高。

美国智库兰德公司（RAND Corporation）在 2017 年的强化美军军力的报告中指出: 中国已成为 美国在东亚地区的假想敌，现在的美国军力不足以 因应和中国的重大军事冲突。由于中国近几年大幅 提升军力, 现在美国对中国的军力比为 2.7: 1, 美 军的军费远远超越中国, 但如果中美两国爆发冲 突, 美军可能在军事对抗中吃下败战。报告中还提 醒美国防部，面对两岸潜在的冲突，如果没有投入 大量的军事投资来强化军力, 周边依赖美军的国 家, 就会失去美国打败中国的信心，而可以促使中 国鼓起勇气掌控东亚的战略环境。

近年来, 由于台湾地区的少数政客为操弄民粹 意识在选举中获得好处, 使得两岸交流停滞或减 缓, 沟通的渠道变少及变小, 彼此的信任度渐渐降 低, 军事风险也慢慢提高, 发生战争的可能性也会 提升。美国学者 Farley（2017）年在国家利益（The National Interest）网站上说明：2018 年最可能爆发第 三次世界大战的地方, 台湾地区排名第二。宋兴洲 指出（2004）：信任是改善两岸彼此关系，促进和 平的重要因素, 而信任是经过双方持续积极的互动 与诚意的交流, 才能加强双方的信任度, 打破双方 因岐见而产生的僵局, 迈向和平稳定的方向前进。 李登辉和陈水扁利用「民粹主义」及「新南向政 策」操弄台湾人民, 让台湾人民产生中国大陆以强 欺弱的虚假信念, 以降低对中国大陆的信任感, 使 其在选举中再次获得政权, 这些都是不顾台湾人民 的福祉, 破坏社会安定的措施 (邓利娟, 2000; 庞 建国，2016）。两岸关系错综复杂，其牵涉到政 治、经济、军事等多方面, 加上国外势力的介入、 政客的操弄及整体经济实力大幅减弱的影响下, 藉 由良性的沟通，提升彼此间的信任度就显的非常重 要。Scott 学者 (2017) 指出早在一千年前亚里斯多 德就提出有效沟通的三大要素: 让人信服 ( ethos), 动之以情 (pathos) 和信之有理 （logos）。两岸的人民如果建立彼此的高忠诚度与 信赖感, 藉由频繁的交流互动连结彼此的情感, 以 
及理性地了解彼此的需求, 军事冲突的风险就会降 低。

本研究从信任博亦来探索两岸间的互动关系, 试着找出双方最适选择及报酬, 藉由影响两岸人民 信任的三个重要因子, 即大陆出台给台湾人民的利 益、台湾回馈给大陆利益, 以及两岸采用「信任」 策略而产生的合作报酬, 建构两岸非零和信任博弯 模型, 经由博弯的纯粹策略均衡分析, 我们发现: 当双方认为采用「信任」策略而产生的合作报酬很 高时, 双方就会走向合作, 都会采用「信任」的策 略, 双方获得的期望报酬都会达到最高, 这结果对 双方是最佳的选择。而双方在信任博弯中, 失去合 作的意愿, 则两岸军事冲突的风险会渐渐提高。我 们综观以上的经济指标及研究报告, 影响两岸信任 关系的因素有三个, 分别是：两岸经济总体差距过 大、台湾地区的少数政客操弄民粹意识, 以及国外 势力恶意介入两岸交流。这三个会降低彼此互信合 作得到的报酬, 因此会降低双方合作的意愿。我们 发现现今两岸僵局正走在一个混合策略的信任博弯 里, 经由数值模拟计算本模型的混合策略均衡后, 发现大陆的混合策略均衡的期望报酬不会受到三个 重要因子影响而改变, 而台湾的混合策略均衡的期 望报酬, 会随着两岸采用「信任」策略的合作报酬 而改变, 当两岸采用「信任」策略的报酬愈高, 两 岸合作的意愿就愈高, 台湾人民在这场博亦中获得 的期望报酬也会愈高。日前中国大陆提出多项惠台 政策, 台湾接受这些惠台政策, 才会提高台湾的混 合策略纳什均衡的期望报酬, 对台湾才是最好的选 择。

\section{2. 信任博亦（Trust Game）}

Berg 等学者在 1995 年提出最著名的投资博亦 ( Investment game) 或称为信任博亦 (Trust game), 来验证参赛者的信任度, 博弯的规则如 下: 在两个单独的 $\mathrm{A}$ 和 $\mathrm{B}$ 房间里各有若干人, 房间 $\mathrm{A}$ 的人有 10 元, 为 $「$ 利益发送者」, 可以将他 / 她 的钱给房间 $\mathrm{B}$ 的人; 房间 $\mathrm{B}$ 的人是「利益接收 者」。在实验的第一阶段, 利益发送者即房间 $\mathrm{A}$ 的 人有权决定给房间 $\mathrm{B}$ 的人多少钱 (范围为 $0-10$ 元）。事先告知每个人，如果房间 $\mathrm{A}$ 中的人决定将 一些钱给房间 B 的人，房间 B 的人收到房间 A 中的 人给的金额会增加三倍, 例如: 房间 $\mathrm{A}$ 中的人拿 3 元给房间 $B$ 的人，则房间 $B$ 的人会得到 $3 \times 3=9$ 元 (3 元以外的获得, 从开放系统的其它资源中得到, 具体金额是博亦设定的实验条件），而 B 房间的人 可以回馈给房间 A 中给他/她的人。第一次实验获 得 30 组配对成功, 实验结果发现: A 房间的 30 个人 中只有 2 人不愿分钱给房间 $\mathrm{B}$ 的人。 $\mathrm{A}$ 房间有 30 个
利益发送者, 他 / 她们平均发送的金额 5.16 美元。 而且, B 房间的 30 个利益接收者中有 11 人收到钱 后, 回馈金额会比 A 房间的利益发送者送给他们的 还多。相反地, 其它 19 人会比 $\mathrm{A}$ 房间的利益发送者 送给他们的还少。第二次实验前先公布第一次实验 的发送金额和回馈金额的平均值，第二次实验结果 发现: 利益发送者分享的金额变多, 利益接收者回 馈的金额也跟着变多。这信任博峦理论的纳什均衡 解是房间 $\mathrm{A}$ 的人不会送任何一毛钱给房间 $\mathrm{B}$ 的人, 但为什么实验的结果是：房间 $\mathrm{A}$ 的人愿意给房间 $\mathrm{B}$ 的人一些钱, 本研究认为是合作互惠的动机使得双 方愿意互利, 互利愈多就会愈来愈信任对方。

我们为什么要关心信任度? 因为信任博弯在现 实生活中被视为有益的东西。例如, 休假时我们把 房子钥匙留给你的邻居, 请他 / 她帮忙照顾一下, 回来带些礼物给你的邻居, 多次后, 他会主动的帮 你照顾室内的植物, 这是彼此提升信任感带来的益 处。Fukuyama 学者 (1995) 认为: 公民之间的信任 度被认为是社会更加繁荣的一个因素。Johnson 和 Mislin (2011) 在研究不同的国家间进行信任博弯 时, 也呈现出类似的结论, 他们分析更为复杂的信 任博弯后发现：撒哈拉以南的非洲地区与北美洲地 区相比的信任程度要小很多。因为两地区里的人民 平均收入和信任度有着相互影响的关系, 人均收入 愈少, 人民彼此信任度就愈低; 人均收入愈高, 信 任度就愈高, 因此, 决策者应将信任程度与经济绩 效挂钩。同时, 信任博亦可能被视为确定信任和诚 信等重要指标的主要工具。

目前两岸信任关系就像以下情境: 麦当劳餐厅 刚进入以「中式食物」为主的市场, 它所卖的汉 堡、薯条及可乐等「西式食物」，一时是无法让习 惯吃「中式食物」（例如：包子、豆浆）的居民接 受, 尤其是早餐吃汉堡, 很多人都不习惯, 麦当劳 为了刺激消费于是想出了一个促销的方法, 打出 8 元低价早餐的口号, 这种促销方式, 初期买的人很 少, 但随着降价让一般人可接受时, 例如 8 元早餐 变成 6 元早餐, 想要买的人就会愈来愈多, 时间久 了, 麦当劳的「西式食物」就慢慢让「中式食物」 为主的居民接受。这情境中, 以「中式食物」为主 的市场就是台湾地区的人民, 台湾人民目前吃的食 物是「维持现状」，而「西式食物」就是「和平统 一」, 中国为了希望台湾人能习惯于「和平统 一」, 于是出台一些优惠政策, 这些政策包含引进 台湾优秀人才就业及鼓励台商来大陆创业等等, 这 些促销策略愈来愈吸引台湾年青人至大陆就业, 而 这些增加两岸交流的政策也渐渐发酵, 让台湾地区 的人民对中国逐渐地产生好感, 而信任感也随之增 加。本研究参考 Gambetta 等学者 (2014) 的信任博 
恋（Trust Game）模型，博栾情境用表 1 的静态信任 博恋矩阵（Normal form）描述。

表 1：静态信任博弯矩阵.

\begin{tabular}{|c|c|c|c|}
\hline & \multicolumn{2}{|c|}{$I_{2}$} \\
\hline & & $s_{21}$ & $s_{22}$ \\
\hline \multirow{2}{*}{$I_{1}$} & $s_{11}$ & $\left(u_{1}\left(s_{11}, s_{21}\right), \quad u_{2}\left(s_{11}, s_{21}\right)\right)$ & $\left(u_{1}\left(s_{11}, s_{22}\right), u_{2}\left(s_{11}, s_{22}\right)\right)$ \\
\hline & $s_{12}$ & $\left(u_{1}\left(s_{12}, s_{21}\right), u_{2}\left(s_{12}, s_{21}\right)\right)$ & $\left(u_{1}\left(s_{12 j}, s_{22}\right), u_{2}\left(s_{12} s_{22}\right)\right)$ \\
\hline
\end{tabular}

此矩阵的解释如下:

(1) 某一博弯 $G$ 有两个玩家, 玩家一 $I_{1}$ 是中国大 陆, 它是「利益发送者」; 玩家二 $I_{2}$ 是台湾, 它是

「利益接收者」。用 $S_{i}$ 记第 $i$ 个玩家的策略集合， $u_{i}$ 记 第 $i$ 个玩家的报酬函数 $u_{i}$, 则两个玩家的策略及策略 互动产生的报酬建构的博弯可形式化地表为 $G=\left\{S_{1}\right.$, $\left.S_{2} ; u_{1}, u_{2}\right\}$ 。

(2) 设 $S_{i j}$ 是 $S_{i}$ 中的第 $j$ 个策略。表 1 中两个玩家 都有相同的两个策略选择：信任和不信任，即 $S_{1}=S_{2}=\left(s_{11}, s_{12}\right)=\left(s_{21}, s_{22}\right)=$ （信任，不信任）。双方事 先都知道对方的策略有两个, 由于是同步出手, 玩 家一不知道玩家二会做那一个决定（策略）; 同样 地, 玩家二也不知道玩家一会做那一个决定（策 略），但是它们能规划在博亦中采用那一个策略。

(3) 玩家 $i$ 的报酬函数是 $S_{1} \times S_{2}$ 到实数集的一个 映射, 即

$$
\begin{aligned}
& u_{i}: S_{1} \times S_{2} \rightarrow R \\
&\left(s_{1 j}, s_{2 k}\right) \rightarrow u_{i}\left(\left(s_{1 j}, s_{2 k}\right),\left(s_{1 j}, s_{2 k}\right)\right) \quad j, k \in 1,2
\end{aligned}
$$

（4）在表 1 中，行是玩家一的 $j$ 策略; 而列是玩 家二的 $k$ 策略，玩家一的报酬为 $u_{1}$, 玩家二的报酬为 $u_{2}$, 两者策略互动的组合 $\left(s_{1 j}, s_{2 k}\right)$ 产生双方的策略报酬 组合 $\left(u_{1}\left(s_{1 j}, s_{2 k}\right), u_{2}\left(s_{1 j}, s_{2 k}\right)\right)$, 建构表 1 报酬矩阵。

中国大陆对台湾地区人民示好，自身必有一定 的利益（payoff），记为 $\pi$, 而赠送给台湾人民的利 益记为 $v$ 。如果台湾人民信任大陆而接收此利益 $v$, 假定利益会放大三倍，则台湾人民获得的利益是 $3 v$, 并回馈给大陆利益 $b$ 。如果大陆与台湾人民是同 时出手, 双方策略互动建构信任博亦矩阵如表 2 所 示。

表 2: 信任博亦矩阵（无利他诱因）

\begin{tabular}{cccc}
\hline & & \multicolumn{2}{c}{ 台湾 } \\
\cline { 3 - 4 } & & 信任 & 不信任 \\
\hline 中国 & 信任 & $\pi-v+b, 3 v-b$ & $\pi-v, 3 v$ \\
大陆 & 不信任 & $\pi, 0$ & $\pi, 0$ \\
\hline
\end{tabular}

这一博弯将会产生以下四种报酬组合:

第一种情况：如果大陆采用「信任」策略, 将 原有的利益 $\pi$ 中, 取出利益 $v$ 给台湾人民，台湾人民选 择「信任」及接收大陆给的利益 $v$, 放大三陪后变 $3 v$, 并接着回馈给大陆报酬 $b$, 则大陆的报酬为 $\pi$ $v+b$ ，而台湾人民获得的报酬为 $3 v-b$ 。
第二种情况：如果大陆采用「信任」策略, 将 原有的利益 $\pi$ 中, 赠送利益 $v$ 给台湾地区人民, 然而, 台湾人民选择「不信任」, 接收大陆送的利益 $v$ 后, 放大三陪后变 $3 v$, 但不愿回馈任何利益给大陆, 则大 陆的报酬为 $\pi-v$, 而台湾人民获的报酬为 $3 v$ 。

第三及第四种情况：看表 1 ，因为大陆采用「不 信任」, 他独占原有的利益 $\pi$, 台湾不管选择信不信 任，台湾的报酬都是零，即得不到任何的好处。

当我们假定 $\pi=10, v=3, b=1$ 时, 由表 2 即可

\begin{tabular}{|c|c|c|c|}
\hline & & \multicolumn{2}{|c|}{ 台湾 } \\
\hline & & 信任 & 不信任 \\
\hline 中国 & 信任 & 8,8 & 7,9 \\
\hline 大陆 & 不信任 & 10,0 & 10,0 \\
\hline
\end{tabular}
得到表3。

无利他诱因博栾矩阵的解, 称为纳什均衡组 合。根据Dixit等学者 (2007) 在Games of Strategy书 中说明：在双人博弯报酬矩阵中，可以应用劣势消 去法, 找出双方的纳什均衡策略。劣势消去法概述 为：给定 (固定) 其它参赛者的策略, 从其中一个 参赛者的策略集合中，剔除报酬差的，找出参赛者 间报酬最高的策略，任一参赛者无诱因单方面偏离 此均衡, 这个均衡稳定状态为参赛者最适反应的策 略组合。

对于表 3 的博亦矩阵而言，应用劣势消去法找出 纳什均衡策略, 其步骤如下:

（1）给定台湾始终采用「信任」策略，大陆选 择「信任」策略获得报酬 8 , 选择「不信任」策略的 报酬为 10 , 因为 10 大于 8 , 所以大陆会采用报酬最高 的「不信任」策略。

（2）给定台湾始终采用「不信任」策略，大陆 选择「信任」策略获得报酬 7 , 选择「不信任」策略 的报酬为 10 , 因为 10 大于 7 , 所以大陆也会采用报酬 最高的「不信任」策略。

（3）给定大陆始终采用「信任」策略，台湾选 择「信任」策略获得报酬 8 , 选择「不信任」策略的 报酬为 9 , 因为 9 大于 8 , 所以台湾会采用报酬最高的 「不信任」策略。

（4）给定大陆始终采用「不信任」策略，台湾 选择「信任」策略获得报酬 0 , 选择「不信任」策略 的报酬也为 0 , 所以台湾同时会采用「不信任」和 「信任」策略。

经由劣势消去法, 参赛者报酬最高的决策便构 成赛局的解（solution of a game）, 由以上可知, 当 信任博亦内双方无利他的诱因时, 纳什均衡组合是 两岸都选择「不信任」的策略, 即 \{不信任, 不信 任 $\}$ ，而均衡的报酬组合为 $(10,0)$ ，大陆的报酬 
为 10 , 而台湾的报酬为 0 。这是信任博弯的均衡解, 双方不会偏离的策略。

当信任博弯内双方有利他的诱因时, 也就是如 果中国大陆与台湾采用「信任」策略时, 假定因为 帮助他人，双方合作都会产生额外的报酬 4 ，从而我 们得到表4的信任博亦报酬矩阵（有利他诱因）。同 样地, 用劣势消去法可以找出这博亦的纳什均衡策 略为 $\{$ 信任, 信任 $\}$, 有了利他及互惠的诱因时, 发现大陆转而选择「信任」策略, 而台湾也选择 「信任」策略, 这时信任博弃的均衡报酬组合为 $(12,12)$ ，双方得到最大报酬。

\begin{tabular}{cccc} 
表 4: 信任博弯数值矩阵 (有利他诱因) \\
\cline { 3 - 4 } & & \multicolumn{2}{c}{ 台湾 } \\
\cline { 3 - 4 } & & 信任 & 不信任 \\
\hline 中国 & 信任 & $\mathbf{8 + 4 , 8 + 4}$ & $7+4,9$ \\
大陆 & 不信任 & $10,0+4$ & 10,0 \\
\hline
\end{tabular}

\section{3. 两岸信任博弯模型}

目前中国大陆与台湾地区的两岸人民互相信任 的情境, 可以将它对应在一场「信任博弯」（Trust Game）的模型中。假设有四个因子建构这场博弯, 如下:

$\pi$ : 大陆具有一利益（payoff）；

$v$ : 大陆为了对台湾地区人民示好, 从 $\pi$ 中取出利 益赠送给台湾地区人民;

$b$ : 台湾人民信任大陆而接收利益 $v$, 并回馈给大 陆的利益; 报酬。

$c$ ：大陆及台湾采用「信任」策略而产生的合作

台湾也可以选择「不信任」不回馈。当大陆或 台湾采用「信任」策略时, 这代表彼此愿意合作, 我们设定 $c$ 是个互惠合作的因子，这因子会对台湾及 大陆自身产生报酬，这因子的报酬值愈大，双方采 用「信任」策略的意愿就愈大。我们假设有国外势 力的介入、政客的民粹（还学文，2016）操弄及两 岸经济总体差距过大, 这三个主要因素会降低合作 报酬 $c$, 因此会降低双方合作的意愿。双方的报酬矩 阵如表5。这时博亦会有以下六种情况:

表 5: 两岸信任博亦报酬矩阵

\begin{tabular}{cccc}
\hline & & \multicolumn{2}{c}{ 台湾 } \\
\cline { 3 - 4 } & & 信任 & 不信任 \\
\hline 中国 & 信任 & $\pi-v+b+c, v-b+c$ & $\pi-v+c, v$ \\
大陆 & 不信任 & $\pi, c$ & $\pi, 0$ \\
\hline
\end{tabular}

（1）如果 $\pi>v>c>b$, 假设 $\pi=4, v=3, c=2$, $b=1$, 代入表 5 , 计算后如表 6 , 利用劣势消去法找出 这博弯的纯粹策略纳什均衡解有两组, 第一组为 \{不信任, 信任 $\}$ 的策略, 双方的均衡报酬组合为
$(4,4)$, 第二组为 $\{$ 不信任, 信任 $\}$ 的策略, 双方 的均衡报酬组合为 $(4,2)$ 分析如下:

\begin{tabular}{cccc} 
表 6: & 两岸信任博弯报酬矩阵 $(\pi>v>c>b)$ \\
\cline { 3 - 4 } & & \multicolumn{3}{c}{ 台湾 } \\
\cline { 3 - 4 } & 信任 & 不信任 \\
\hline 中国 & 信任 & $\mathbf{4 , 4}$ & 3,3 \\
大陆 & 不信任 & $\mathbf{4 , 2}$ & 4,0 \\
\hline
\end{tabular}

大陆赠送给台湾地区人民的利益 $v$, 大于大陆及 台湾采用「信任」策略而产生的合作报酬 $c$, 并大于 台湾回馈给大陆利益 $b,\{$ 信任, 信任 $\}$ 的策略组合 和 $\{$ 不信任, 信任 $\}$ 的策略组合都是纯粹策略纳什 均衡解, 两组的报酬为 $(4,4)$ 及 $(4,2)$ 。不管大 陆选择「信任」或「不信任」的策略, 报酬都是 4 , 由于, 台湾只能选择「信任」的策略, 台湾的报酬 会随着大陆的选择而改变, 当大陆选择「信任」, 台湾获得的报酬为 4 , 而大陆选择「不信任」的策 略, 台湾的报酬会降为 2 , 因此, 这博弯的主导权在 中国大陆。

（2）如果 $\pi>v>b>c$ ，假设 $\pi=4, v=3, b=2$, $c=1$, 代入表 5 , 计算后如表 7 , 利用劣势消去法找出 这博弯的纯粹策略纳什均衡解为 \{不信任, 信任 $\}$ 的策略, 双方的报酬组合为 $(4,1)$, 分析如下:

表 7: 两岸信任博弯报酬矩阵 $(\pi>v>b>c)$

\begin{tabular}{cccc}
\hline & & \multicolumn{2}{c}{ 台湾 } \\
\cline { 2 - 3 } & 信任 & 不信任 \\
\hline 中国 & 信任 & 4,2 & 2,3 \\
大陆 & 不信任 & $\mathbf{4 , 1}$ & 4,0 \\
\hline
\end{tabular}

大陆赠送给台湾人民的利益为 $v$ ，大于台湾回馈 给大陆利益 $b$, 并大于台湾及大陆采用「信任」策略 而产生的合作报酬c。大陆采用「不信任」的策略, 而台湾选择「信任」的策略, 是这博弯的纯粹策略 纳什均衡, 由于大陆怕台湾选择「不信任」的策 略, 他的报酬会降为 2 , 因此, 大陆会选择「不信 任」的策略, 而台湾会选择「信任」的策略。

（3）如果 $\pi>c>v>b$, 假设 $\pi=4, c=3, v=2$, $b=1$, 代入表 5 , 计算后如表 8 , 利用劣势消去法找出 这博亦的纯粹策略纳什均衡解为 $\{$ 信任, 信任 $\}$ 的 策略, 双方的报酬组合为 $(6,4)$, 分析如下:

\begin{tabular}{cccc} 
表 8: & 两岸信任博亦报酬矩阵 $(\pi>c>v>b)$ \\
\cline { 3 - 4 } & & \multicolumn{2}{c}{ 台湾 } \\
\cline { 3 - 4 } & 信任 & 不信任 \\
\hline 中国 & 信任 & $\mathbf{6 , 4}$ & 5,2 \\
大陆 & 不信任 & 4,3 & 4,0 \\
\hline
\end{tabular}

如果双方认为采用「信任」策略而产生的合作 报酬很高时, 双方就会走向合作, 都会采用「信 任」的策略, 双方的报酬都会达到最高, 这结果对 
双方是最佳的选择。相反地, 如果双方停止对话, 没有沟通的渠道，彼此认为采用「信任」的策略会 得到较低的合作报酬时，就会走向第2种的情况，大 陆会采取「不信任」的策略。

（4）如果 $\pi>c>b>v$ ，假设 $\pi=4 ， c=3 ， b=2$ ， $v=1$, 代入表 5 , 计算后如表 9 , 利用劣势消去法找出 这博峦的纯粹策略纳什均衡解为 $\{$ 信任, 信任 $\}$ 的 策略, 双方的报酬组合为 $(8,2)$, 分析如下:

表 9: 两岸信任博峦报酬矩阵 $(\pi>c>b>v)$

\begin{tabular}{cccc}
\hline & \multicolumn{2}{c}{ 台湾 } \\
\cline { 3 - 4 } & & 信任 & 不信任 \\
\hline 中国 & 信任 & $\mathbf{8 , 2}$ & 6,1 \\
大陆 & 不信任 & 4,3 & 4,0 \\
\hline
\end{tabular}

这博栾的均衡解和第3种情况一样，如果双方认 为采用「信任」策略而产生的合作报酬很高时，双 方就会走向合作，都会采用「信任」的策略，双方 的报酬为都会达到最高。

（5）如果 $\pi>b>c>v$, 假设 $\pi=4, b=3, c=2$, $v=1$, 代入表 5 , 计算后如表 10 , 利用劣势消去法找 出这博弯的纯粹策略纳什均衡解为 \{信任, 不信 任 $\}$ 的策略。双方的报酬组合为 $(5,1)$, 分析如 下:

表 10: 两岸信任博亦报酬矩阵 $(\pi>b>c>v)$

\begin{tabular}{cccc}
\hline & & \multicolumn{2}{c}{ 台湾 } \\
\cline { 3 - 4 } & & 信任 & 不信任 \\
\hline 中国 & 信任 & 8,0 & $\mathbf{5 , 1}$ \\
大陆 & 不信任 & 4,2 & 4,0 \\
\hline
\end{tabular}

台湾回馈给大陆利益 $b$, 大于台湾及大陆采用 「信任」策略而产生的合作报酬 $c$ ，并大于大陆赠送 给台湾地区人民的利益 $v$ 。这博弯的最适结果为：大 陆采用「信任」的策略, 而台湾选择「不信任」的 策略，虽然大陆采用「信任」策略会得到最高的报 酬8，但是台湾并不会采用「信任」的策略，而是采 取「不信任」的策略，不回馈给大陆任何利益，反 而会得到 1 的报酬。这博弯的均衡结果有趣的地方 是：只要台湾回馈给大陆利益 $b$ 跟 $c$ 和 $v$ 比, 是最高的 值，而双方因合作产生的报酬 $c$ ，又大于 $v$ 时，虽然大 陆知道台湾会选择「不信任」的策略, 但因为双方 认为合作可以产生的报酬 $c$ ，大于大陆赠送给台湾地
区人民的利益为 $v$ 时，大陆还是会选择「信任」的策 略。

（6）如果 $\pi>b>v>c$ ，假设 $\pi=4 ， b=3 ， v=2$, $c=1$, 代入表 5 , 计算后如表 11 , 利用劣势消去法无 法找出这博峦的纯粹策略纳什均衡解, 分析如下:

表 11：两岸信任博弯报酬矩阵 $(\pi>b>v>c)$

\begin{tabular}{cccc}
\hline & \multicolumn{2}{c}{ 台湾 } \\
\cline { 3 - 4 } & & 信任 & 不信任 \\
\hline 中国 & 信任 & 6,0 & 3,2 \\
大陆 & 不信任 & 4,1 & 4,0 \\
\hline
\end{tabular}

台湾回馈给大陆利益 $b$ 大于大陆赠送给台湾地区 人民的利益 $v$, 并大于台湾及大陆采用「信任」策略 而产生的合作报酬 $c$ 。这情况利用劣势消去法找不到 博弯的纯粹策略纳什均衡解, 根据Lemke和Howson 学者（1964）说明一个博峦, 如果没有纯粹策略均 衡，必定有一个混合策略均衡，我们把表4转换成表 11 为两岸混合策略信任博弯。假设中国选择「信 任」策略的概率为 $p$, 选择「不信任」策略的概率为 $1-p$, 台湾选择「信任」混合策略的期望报酬为 $(v-b+$ $c) p+c(1-p)$ ，选择「不信任」混合策略的期望报酬 为 $v p+0(1-p)$ （如表 12），我们将两者混合策略的期 望报酬相等，可以计算 $p$ 值（A. Dixit \& S. Skeath， 2011），如下:

$$
\left\{\begin{array}{l}
(v-b+c) p+c(1-p)=v p+0(1-p) \\
p=c / b
\end{array}\right.
$$

假设台湾地区选择「信任」策略的概率为 $q$, 则 选择「不信任」策略的概率为 1- $q$, 中国选择「信 任」混合策略的期望报酬为 $(\pi-(v-b)+\mathrm{c}) q+(\pi-v+c)(1-$ $q)$, 选择「不信任」混合策略的期望报酬为 $\pi q+\pi(1-q)$ （如表 12 ），我们将两者混合策略的期望报酬相 等, 同样可以计算 $q$ 值 (A. Dixit \& S. Skeath, 2011），如下:

$$
\left\{\begin{array}{l}
(\pi-(v-b)+c) q+(\pi-v+c)(1-q)=\pi q+\pi(1-q) \\
q=(v-c) / b
\end{array}\right.
$$


表 12: 两岸信任博弯混合策略报酬矩阵 $(\pi>b>v>c)$

\begin{tabular}{ccccc}
\hline & & \multicolumn{2}{c}{ 台湾 } & $\boldsymbol{q}$-mix \\
\cline { 3 - 5 } & & 信任 $\boldsymbol{q}$ & 不信任 1-q & $(\pi-(v-b)+\mathrm{c}) q+(\pi-v+c)(1-q)$ \\
中国大 & 信任 $\boldsymbol{p}$ & $\pi-v+b+c, v-b+c$ & $\pi-v+c, v$ & $\pi q+\pi(1-q)$ \\
陆 & 不信任 1-p & $\pi, c$ & $\pi, 0$ & \\
\hline & $\boldsymbol{p}$-mix & $(v-b+c) p+c(1-p)$ & $v p+0(1-p)$ & \\
\hline
\end{tabular}

求出此博弯的混合策略纳什均衡为: $p=c / b ; q=(v$ $c) / b$ 或以 $(c / b,(v-c) / b)$ 表示。

在这博弯中，我们用Gibbon（1992）的方法, 计算中国的混合策略纳什均衡的期望报酬为式(3)所 示。

$$
\left\{\begin{array}{l}
E_{\text {大陆 }}=(\pi-(v-b)+c) p q+(\pi-v+c) p(1-q)+\pi(1-p) q+\pi(1-p)(1-q) \\
E_{\text {大陆 }}=b p q-v p+c p+\pi
\end{array}\right.
$$

将方程式(1)及(2)代入方程式(3), 我们得到

$$
\left\{\begin{array}{l}
E_{\text {大陆 }}=b(c / b)((v-c) / b)-v(c / b)+c(c / b)+\pi \\
E_{\text {大陆 }}=\pi
\end{array}\right.
$$

这结果非常有趣, 当 $\pi>b>v>c$ 时, 双方采取随 机的混合策略，不管其它变量 $b 、 v 、 c$ ，是如何？大 陆在这博峦的混合策略纳什均衡的期望报酬是原来 假设的利益（payoff） $\pi$ 。

而台湾的混合策略纳什均衡的期望报酬为:

$$
\left\{\begin{array}{l}
E_{\text {台湾 }}=(v-b+c) p q+v p(1-q)+c(1-p) q+0(1-p)(1-q) \\
E_{\text {台湾 }}=v p+c p-b p q
\end{array}\right.
$$

将方程式(1)及(2)代入方程式(5)得到

$$
\left\{\begin{array}{l}
E_{\text {台湾 }}=v(c / b)+c((v-c) / b)-b(c / b)((v-c) / b) \\
E_{\text {台湾 }}=(c v) / b
\end{array}\right.
$$

如果将 $\pi=4, b=3, v=2, c=1$, 代入方程式 (1)(2), 得到中国大陆及台湾选择「信任」策略的概 率, 分别是 $p=1 / 3 ; q=1 / 3$ 。

再将上式代入方程式(4)(6), 得到中国大陆及台 湾的混合策略纳什均衡的期望报酬, 分别是 $E_{\text {大陆 }}=4$; $E_{\text {台湾 }}=2 / 3$ 。

\section{4. 数值模拟与讨论}

由于本研究提出博弯的第一到第五种情况, 可 以找出纯粹策略纳什均衡, 利用数值分析无法清楚 地观察到台湾期望报酬和影响因子 $v, c, b$ 之间的变 化。但是在第六种情况下是属于随机混合策略博 栾, 我们可以经由数值模拟的方式, 找出混合策略 纳什均衡, 来验证两岸信任博弯模型的可行性, 四
个因子的数值设定范围为: $\pi=120 ; 119 \geq b \geq 81$; $80 \geq v \geq 41 ; 40 \geq c \geq 1$, 代入方程式(6)计算台湾的混合策 略纳什均衡期望值, 观察它和 $v, c, b$ 之间的关系。

\section{1. 可信度分析}

本研究提出的信任博弯模型, 在第六种情况 下，不管其它影响因子 $v, c, b$ 的数量是如何? 大陆 在这博弯的混合策略均衡的期望报酬都是原来假设 的利益（payoff） $\pi$, 所以我们只分析台湾混合策略

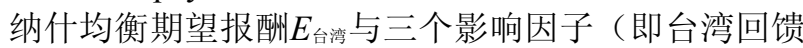
给大陆利益 $b$ 、大陆赠送给台湾地区人民的利益 $v$ ，以 及双方采用「信任」策略合作报酬 $c$ ）之间的互相影 响关系, 并制成图 2 至 4, 以便于验证本研究提出混 合策略纳什均衡的可信度。

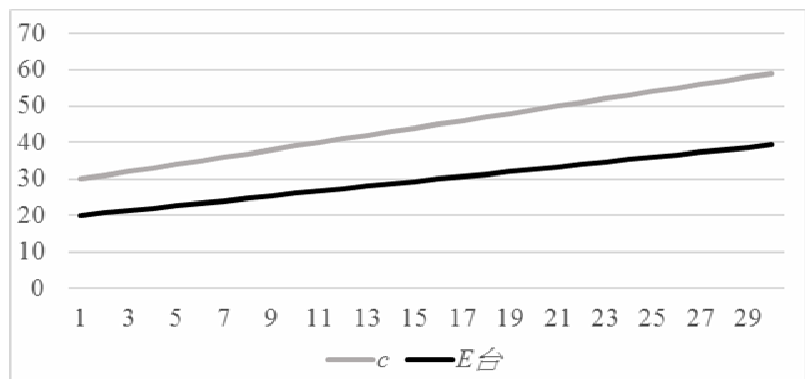

图 2: 双方采用「信任」策略合作报酬和台湾的混合策略 纳什均衡期望报酬关系

（1）双方采用「信任」策略报酬 $c$ 与台湾混合

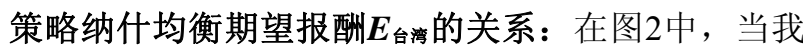
们将台湾回馈给大陆利益 $b$ 及大陆赠送给台湾地区人 民的利益 $v$ 设为固定值, 然后逐渐增加双方采用「信 任」策略报酬 $c$ 的数值，发现台湾混合策略纳什均衡 期望报酬逐渐上升，它代表双方认为彼此信任的报 酬增加, 双方合作的意愿逐渐增强, 而台湾混合策 略纳什均衡的期望报酬也会逐渐增加，双方采用 「信任」策略报酬愈高, 对于台湾愈有利。 


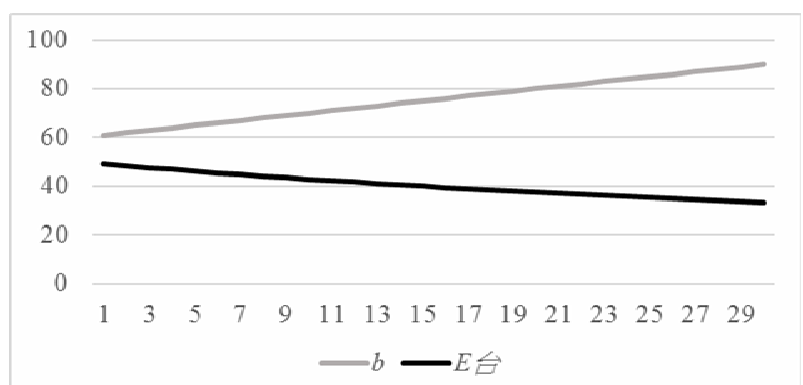

图 3: 台湾回馈给大陆利益和台湾的混合策略纳什均衡期 望报酬关系

（2）台湾回馈给大陆利益 $\boldsymbol{b}$ 与台湾混合策略纳

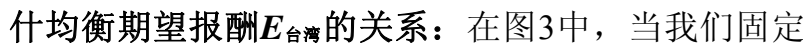
大陆赠送给台湾地区人民的利益 $v$ 及双方采用「信 任」策略合作报酬 $c$ 的数值, 把两者设为固定值, 然 后逐渐增加台湾回馈给大陆利益 $b$ 的数量, 我们可以 发现台湾混合策略纳什均衡期望报酬会随之下降。 当双方在进行这场信任博弯时, 台湾回馈给大陆利 益 $b$ 是高于 $v$ 和 $c$ 因子（即大陆赠送给台湾地区人民的 利益及双方采用「信任」策略合作报酬），台湾回 馈给大陆利益愈多, 台湾得到的期望报酬就愈少, 相对地, 台湾回馈给大陆利益愈少, 台湾得到的期 望报酬就愈高。

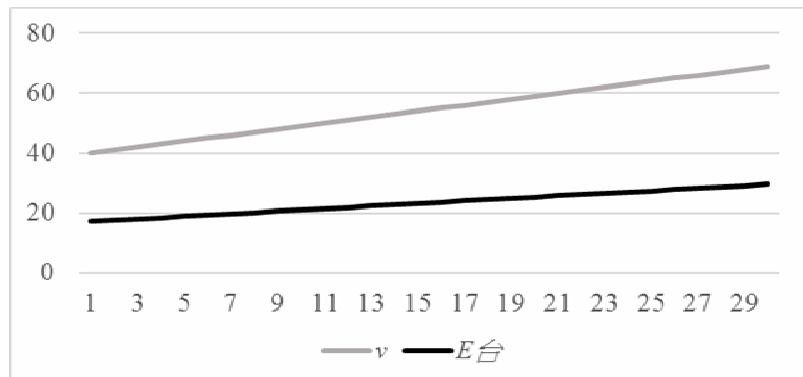

图4: 大陆赠送的利益和台湾的混合策略纳什均衡期望报 酬关系

（3）大陆赠送给台湾地区人民的利益 $v$ 与台湾

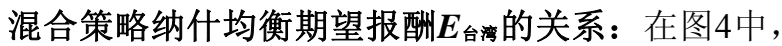
当我们将台湾回馈给大陆利益 $b$ 、双方采用「信任」 策略合作报酬 $c$ 设为固定值, 然后逐渐增加大陆赠送 给台湾地区人民的利益 $v$ 的数值, 发现台湾混合策略 纳什均衡期望报酬逐渐上升, 如图4, 它代表大陆赠 送给台湾地区人民的利益愈多，而台湾混合策略纳 什均衡的期望报酬也会逐渐增加; 相反地, 大陆赠 送给台湾地区人民的利益愈少, 台湾获得的报酬就 愈少。

由以上三点, 我们可以知道双方采用「信任」 策略合作报酬和大陆赠送给台湾地区人民的利益, 两者跟台湾的混合策略纳什均衡期望报酬 $E$ 台的关 系成正比, 也就是说, 台湾的混合策略纳什均衡期 望报酬值会随着这两个因子的数值增加而增加, 并
随着两个因子的数值减少而减少。而台湾回馈给大 陆利益 $b$ 与台湾的混合策略纳什均衡期望报酬值的 关系成反比, 台湾的混合策略纳什均衡期望报酬值 会随着这个因子的数值增加而减少。

我们进一步分析两岸信任博弯模型的第六种情 况, 在 $\pi>b>v>c$ 的前提下, 藉由逐渐递增或递减四

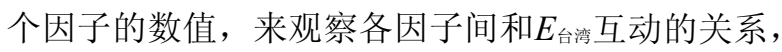
我们汇集以下四种实验结果, 根据结果分别对应实 际现况予以分析。

(1) $c$ 的值固定，递增或递减 $b$ 和 $v$ 的值，来观

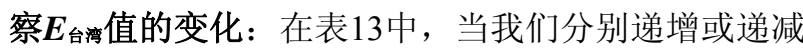

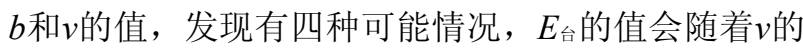
值递增而递增, 或 $v$ 的值递减而随之递减, 并不会受 到 $b$ 值所影响。我们可以解读: 当我们固定双方采用 「信任」策略合作报酬 $c$ 的值时, 台湾的混合策略纳

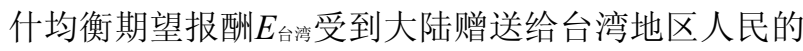
利益 $v$ 的影响较高。

\begin{tabular}{ccccc} 
表 13: & $b 、 v$ 值两者与 $E_{\text {台湾值的变化 }}$ \\
\hline 项次 & $b$ & $v$ & $c$ & $E_{\text {台 }}$ \\
\hline 1 & 递增 & 递增 & 固定 & 递增 \\
2 & 递增 & 递减 & 固定 & 递减 \\
3 & 递减 & 递增 & 固定 & 递增 \\
4 & 递减 & 递减 & 固定 & 递减 \\
\hline
\end{tabular}

（2） $b$ 的值固定，递增或递减 $v$ 和 $c$ 的值，来观 察 $\boldsymbol{E}$ 台浮值的变化: 在表14中, 当我们分别递增或递减

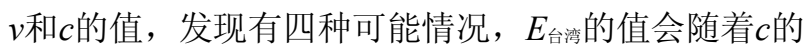
值递增而递增，以及 $c$ 的值递减而随之递减，并不会 受到 $v$ 值所影响。我们可以解读: 当我们固定台湾回 馈给大陆利益 $b$ 的值时, 台湾的混合策略纳什均衡期 望报酬 $E$ 台浮受到双方采用「信任」策略合作报酬 $c$ 的 影响较高。

表 14: $v 、 c$ 值两者与 $E$ 台浮值的变化

\begin{tabular}{ccccc}
\hline 项次 & $b$ & $v$ & $c$ & $E_{\text {台 }}$ \\
\hline 1 & 固定 & 递增 & 递增 & 递增 \\
2 & 固定 & 递增 & 递减 & 递减 \\
3 & 固定 & 递减 & 递增 & 递增 \\
4 & 固定 & 递减 & 递减 & 递减 \\
\hline
\end{tabular}

（3） $v$ 的值固定，递增或递减 $b$ 和 $c$ 的值，来观

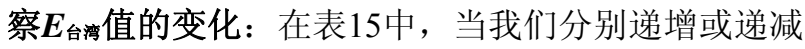

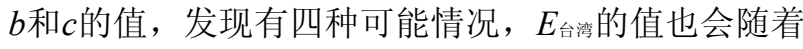

\begin{tabular}{|c|c|c|c|c|}
\hline 项次 & $b$ & $v$ & $c$ & $E_{\text {台湾 }}$ \\
\hline 1 & 递增 & 固定 & 递增 & 递增 \\
\hline 2 & 递增 & 固定 & 递减 & 递减 \\
\hline 3 & 递减 & 固定 & 递增 & 递增 \\
\hline 4 & 递减 & 固定 & 递减 & 递减 \\
\hline
\end{tabular}

$c$ 的值递增而递增, 或 $c$ 的值递减而随之递减, 并不会 受到 $b$ 值所影响。我们可以解读: 同样地, 台湾的混 
合策略纳什均衡期望报酬与双方采用「信任」策略 合作报酬 $c$ 的互相影响较大。

(4) 经由递增或递减 $b 、 v$ 和 $c$ 的值，来观察 $E_{\text {台湾 }}$ 值的变化: 在表 16 中, 当我们分别递增或递减 $v 、 b$

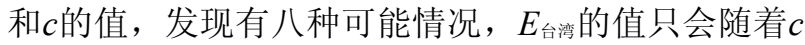
的值递增而递增，或 $c$ 的值递减而随之递减，并不会 受到 $b$ 和 $v$ 值所影响。我们可以解读: 同样地, 就算三 个因子同时变动，台湾的混合策略纳什均衡期望报 酬只和双方采用「信任」策略的合作报酬 $c$ 的互相影 响较大。

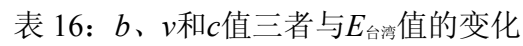

\begin{tabular}{lcccc}
\hline $\begin{array}{l}\text { 项 } \\
\text { 次 }\end{array}$ & $b$ & $v$ & $c$ & $E_{\text {台湾 }}$ \\
\hline 1 & & & & \\
2 & 递增 & 递增 & 递增 & 递增 \\
3 & 递增 & 递减 & 递减 \\
4 & 递增 & 递减 & 递增 & 递增 \\
5 & 递减 & 递减 \\
6 & 递减 & 递增 & 递增 & 递增 \\
7 & 递减 & 递减 & 递增 & 递增 \\
8 & 递减 & 递减 & 递减 & 递减 \\
\hline
\end{tabular}

从这场 $\pi>b>v>c$ 的混合策略信任博弯的实验的 结果来看, 不管 $b$ 和 $v$ 值递增或递减的变动, 台湾的混

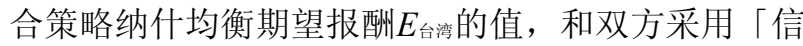
任」策略报酬 $c$ 有较强的互动影响关系, 台湾的混合 策略纳什均衡期望报酬会随着, 双方采用「信任」 策略的合作报酬 $c$ 的增加而增加，随着它减少而减 少。

\section{2. 讨论}

两岸长期以来，有三个因素影响着彼此间的信 任度, 分别是: 两岸经济总体差距过大、台湾少数 政客操弄民粹意识及外国势力恶意介入两岸关系。
这三个主要因素会降低双方采用「信任」策略的合 作报酬, 第一项两岸经济总体差距变小, 对两岸互 信的影响已渐渐消失。第二及第三项是现在影响两 岸互信最大的阻力。但根据中国大陆不断的宣布多 项惠台措施, 提供两岸互信的助力, 其出台政策主 要目的是让台湾人民感受到中国大陆的善意, 强化 两岸的信任感，提升双方的合作意愿。两岸人民信 任的意向正在被阻力和助力拉扯中, 本研究分析出 两岸信任博峦纯粹策略纳什均衡时（如表 17），发 现：当两岸信任度很低时（即双方采用「信任」策 略报酬很低），在第一、二及第五种情况时，双方 的互惠互利的意愿会走下坡, 双方得不到较高的报 酬或利益。唯有信任度很高时，在第三及四的情况 里, 两岸才会迈向经济繁荣、和平稳定的方向前 进。

接着分析信任模型的第六种情况, 即 $\pi>b>v>$ $c$ ，这和现行两岸互惠、互信关系低迷的情况类似。 在这信任博峦里, 台湾回馈给大陆的利益 $b$ 大于大 陆赠送给台湾地区人民的利益 $v$, 并大于台湾及大陆 采用「信任」策略而产生的合作报酬 $c$ ，也就是两岸 认为采用「信任」策略而产生的合作报酬是所有影 响因子最低。本研究经由四组数值模拟实验, 分析 信任博变的混合策略纳什均衡（如表 13-16），发现 影响台湾混合策略纳什均衡期望报酬最大的因子, 还是两岸互信的合作报酬 $c$, 所以根据两岸的地缘 及同文同种的特性，台湾面对大陆接二接三的惠台 政策，只有朝向互信、互惠的方向前进，才能取得 自身最大报酬及利益。

最后, 在表 17 中分析六种情况的信任博峦纳什 均衡汇总结果, 我们发现第一、二及第五种情况, 双方采用「信任」策略而产生的合作报酬 $c$ 太低，这 信任博弯走下去, 无法让双方达到互信的结果。目 前台湾当局不承认九二共识, 加上国外势力破坏两 岸关系, 使得两岸合作互信的报酬最低, 于是两岸

表 17: 两岸信任博弯分析六种情况的纳什均衡汇总

\begin{tabular}{ccccccc}
\hline 项次 & $\mathbf{1}$ & $\mathbf{2}$ & $\mathbf{3}$ & $\mathbf{4}$ & $\mathbf{5}$ & $\mathbf{6}$ \\
\hline 情况 & $\pi>v>c>b$ & $\pi>v>b>c$ & $\pi>c>v>b$ & $\pi>c>b>v$ & $\pi>b>c>v$ & $\pi>b>v>c$ \\
\hline $\begin{array}{c}\text { 大陆均 } \\
\text { 衡策略 }\end{array}$ & $\begin{array}{c}\text { 信任 (或不 } \\
\text { 信任) }\end{array}$ & 不信任 & 信任 & 信任 & 信任 & 选择「信任」的混合策略均衡概率 \\
\hline $\begin{array}{c}\text { 台湾均 } \\
\text { 衡策略 }\end{array}$ & 信任 & 信任 & 信任 & 信任 & 不信任 & 选择「信任」的混合策略均衡概率 \\
\hline $\begin{array}{c}\text { 大陆均 } \\
\text { 衡报酬 }\end{array}$ & 4 & 4 & 6 & 8 & 5 & $\mathbf{q}^{\prime / / 3}$ \\
\hline $\begin{array}{c}\text { 台湾均 } \\
\text { 衡报酬 }\end{array}$ & $4(2)$ & 1 & 4 & 2 & 1 & $E_{\text {台 }=4}$ \\
\hline
\end{tabular}


走向信任博弯的第六种情况, 在这混合策略博栾 中, 双方选择「不信任」的混合策略均衡概率 (1$p=2 / 3,1-q=2 / 3$ ), 都是 $2 / 3=0.667$, 比选择「信任」 的混合策略均衡概率 (1/3) 还高, 因此双方军事冲 突的风险就会提高。然而中国大陆提出多项惠台政 策, 如图 4 分析的结果, 台湾接受这些惠台政策, 才会提高台湾混合策略纳什均衡的期望报酬, 对台 湾才是最好的选择。

\section{5. 结论与未来研究}

海峡两岸中国人分治快 70 年, 自从 1949 年中华 人民共和国成立，中华民国政府转移至台湾地区， 两岸人民的关系就像有着深仇大恨的兄弟互不往 来。随着时光流逝, 彼此的恨意也渐渐消失在这时 空的洪流中，但彼此间的冲突，时有时无的会被挑 起, 造成两岸人民利益的损失。自从 1987 年两岸开 放探亲以来, 双方已进入了一场信任博亦, 直到现 在, 这场博弯还持续进行着。然而, 台湾少数政客 操弄民粹意识及外国势力恶意介入两岸合作的阻 力, 不断地降低台湾采用「信任」策略而产生的合 作报酬, 并影响台湾跟中国大陆的合作意愿。本研 究在提出的信任博弯模型中, 找出六种可能情况的 纳什均衡解, 发现两岸信任度很低时, 即双方采用 「信任」策略报酬小于台湾回馈给大陆的利益及大 陆赠送给台湾地区人民的利益时，只有不断地强化 彼此的信任度, 增加合作的报酬, 台湾的报酬及利 益才会增加, 如果两岸信任合作的报酬渐渐增加, 就会达到双贏的均衡结果, 降低军事冲突的风险。

未来研究希望藉由抽样问卷调查及搜集两岸與 情数据, 来实际验证本模型的有效性。

\section{参考文献}

[1] 邓利娟. 浅析台湾政局变化对经济发展的影响.台湾研 究集刊[J]. 2000.(2):37-42.

[2] 还学文. 到底什么是「民粹」? 重新认识一个被误解和 滥用的词汇,2016,见:

https:/gushi.tw/\%E5\%88\%B0\%E5\%BA\%95\%E4\%BB $\% 80$ $\% \mathrm{E} \% \mathrm{BA} \% \mathrm{BC} \% \mathrm{E} 6 \% 98 \% \mathrm{AF} \% \mathrm{E} 3 \% 80 \% 8 \mathrm{C} \% \mathrm{E} 6 \% \mathrm{~B} 0 \% 91$

$\%$ Е7\%B2\%B9\%Е3\%80\%8D\%ЕF\%BC\%9F\%Е9\%87\%8D $\%$ E6\%96\%B0\%E8\%AA\%8D\%E8\%AD\%98\%E4\%B8\%80 $\%$ E5\%80\%8B\%E8\%A2\%AB\%E8\%AA\%A4\%E8\%A7\%A3 $\% \mathrm{E} \% 92 \% 8 \mathrm{C} \% \mathrm{E} 6 \% \mathrm{BF} \% \mathrm{AB} \% \mathrm{E} 7 \% 94 \% \mathrm{~A} 8 /$

[3] 宋兴洲.两岸关系的突破: 从风险、信任到和解.全球 政治评论,2004,(6):35-74.

[4] 庞建国. 从经济效益看两岸关系,台海研究,2016,(3):5764.

[5] An Interactive Look at the U.S.-China Military Scorecard, 2017 , https://www.rand.org/paf/projects/us-china-scorecard. html.

[6] J. Berg, J. Dickhaut and K. McCabe, Trust, reciprocity, and social-history, Games and Economic Behavior, 10(1)(1995)122-142.

[7] T. Callen, Gross Domestic Product: An Economy's All, FINANCE \& DEVELOPMENT, 2017,

http://www.imf.org/external/pubs/ft/fandd/basics/gdp.htm.

[8] O. David, A. W. Peter, A. Brenna, S. M. John, C. P. Carter, U.S. Military Capabilities and Forces for a Dangerous World Rethinking the U.S. Approach to Force Planning, RAND Corporation, 2017,

https://www.rand.org/pubs/research_reports/RR1782.html.

[9] A. Dixit and Skeath S. Games of Strategy, W. W. Norton \& Company, 2007, p574.

[10]R. Farley, 5 Places World War III Could Start in 2018, The National Interest, http://nationalinterest.org/blog/thebuzz/5-places-world-war-iii-could-start-2018-23686.

[11] J. Florence, K. Ksenia, and C. S. Sweta, Impact of migration on income levels of in advanced economies, Spillover notes (International Monetary Fund), 2016:8.

[12]F. Fukuyama, Trust: The Social Virtues and the Creation of Prosperity, New York: Free Press. 1995, p457.

[13]D. Gambetta and W. Przepiorka, Natural and Strategic Generosity as Signals of Trustworthiness. PLoS ONE], 9(5)(2014) e97533. https://doi.org/10.1371/journal.pone.0097533.

[14] R. Gibbon, A Primer in Game Theory. Harvester Wheatsheaf, 1992.

[15] IMF: World Economic and Financial Surveys, The World Economic Outlook (WEO) database,2017, https:/www.imf.org/external/pubs/ft/weo/2017/02/weoda ta/index.aspx.

[16] A. Johnson and A. Mislin, Trust games: A meta-analysis. Journal of Economic Psychology, 32(2011)865-889.

[17] S. L. Kastner, Is the Taiwan strait still a flash point? rethinking the prospects for armed conflict between china and Taiwan. International Security, 40(3)(2015)54-92.

[18] C. E. Lemke, and J. T. Howson, Equilibrium Points of Bimatrix Games, SIAM J. Applied Math, 12(1964)413423.

[19]F. Raymond and M. Francois, A game theory view of military conflict in the Taiwan strait, Defense \& Security Analysis, 19(4) (2003)327-348.

[20]E. Scott, Three Elements of Great Communication, According to Aristotle Howard Business Review, https://hbr.org/2013/01/three-elements-of-greatcommunication-according. 